\title{
Electronic and magnetic properties of $\mathrm{NiS}_{2}, \mathrm{NiSSe}$ and $\mathrm{NiSe}_{2}$ by a combination of theoretical methods
}

\author{
Cosima Schuster, ${ }^{1}$ Matteo Gatti, ${ }^{2}$, and Angel Rubio ${ }^{2}$ \\ ${ }^{1}$ Institut für Physik, Universität Augsburg, D-86135 Augsburg, Germany \\ ${ }^{2}$ Nano-Bio Spectroscopy group and ETSF Scientific Development Centre, \\ Dpto. Física de Materiales, Universidad del País Vasco, \\ Centro de Física de Materiales CSIC-UPV/EHU-MPC and DIPC, Av. Tolosa 72, E-20018 San Sebastián, Spain
}

(Dated: February 28, 2012)

\begin{abstract}
We investigate the electronic and magnetic properties of $\mathrm{NiS}_{2}$, which, by varying the chemical composition substituting $\mathrm{S}$ by Se atoms or applying pressure, can be driven across various electronic and magnetic phase transitions. By combining several theoretical methods, we highlight the different role played by the chalcogen dimers and the volume compression in determining the phase transitions, through variations of the chalcogen $p$ bonding-antibonding gap, the crystal-field splitting and the broadening of the bandwidths. While the generalized gradient approximation (GGA) of density-functional theory fails to reproduce the insulating nature of $\mathrm{NiS}_{2}$, it describes well the magnetic boundaries of the phase diagram. The large GGA delocalization error is corrected to a large extent by the use of GGA $+\mathrm{U}$, hybrid functionals or the self-consistent COHSEX $+\mathrm{GW}$ approximation. We also discuss the advantages and the shortcomings of the different approximations in the various regions of the phase diagram of this prototypical correlated compound.

PACS numbers: 73.20.-r, 74.25.Jb, 85.25.Am
\end{abstract}

\section{INTRODUCTION}

The crystal-field splitting, the onsite Coulomb interaction $\mathrm{U}$, and the $3 d$ bandwidth $W$ are almost equal in the pyrites $\mathrm{MX}_{2}(\mathrm{M}=\mathrm{Fe}, \mathrm{Co}, \mathrm{Ni}, \mathrm{Cu}, \mathrm{Zn} ; \mathrm{X}=\mathrm{S}$ or $\mathrm{Se})$, which gives rise to a large variety of electrical, magnetic and optical properties in these compounds. Of special interest is the insulator-metal transition (IMT) in $\mathrm{NiS}_{2}$ with Se doping or under pressure. The transition is not accompanied by a change in the lattice symmetry and is commonly believed to be driven by the electron-electron interactions ${ }^{1,2}$. Thus $\mathrm{NiS}_{2}$ would be a typical strongly correlated insulator. In the Mott-Hubbard picture, application of pressure or the modification of the chemical composition are in fact two equivalent ways of controlling the bandwidth $\mathrm{W}$, keeping $\mathrm{U}$ unchanged. In both cases reducing the U/W ratio leads to the IMT. Substitution of $\mathrm{S}$ with Se in the antiferromagnetic insulator $\mathrm{NiS}_{2}$ makes the low-temperature $\mathrm{NiS}_{2-x} \mathrm{Se}_{x}$ phase diagram quite complex ${ }^{2}$. Not only the IMT takes place at $x=0.44$, but at $x=1$ there is an additional magnetic transition to a paramagnetic phase. This is in contrast to $\mathrm{NiS}_{2}$ under pressure, where the electronic IMT and the magnetic transition occur at the same time (at $\mathrm{P}=2.9$ $\left.\mathrm{GPa}^{3}\right)$.

UV and x-ray photoemission, which are among the most prominent tools to probe the electronic structure of materials, are surface sensitive techniques. But in $\mathrm{NiS}_{2-x} \mathrm{Se}_{x}$ the surface electronic structure is known to be very different compared to the bulk ${ }^{4}-\underline{6}$ (in $\mathrm{NiS}_{2}$ also surface magnetism is significantly different from bulk magnetism ${ }^{7}$ ). In fact, for $\mathrm{NiS}_{2}$ even if optical measurements find a $0.3 \mathrm{eV}$ optical gap $\stackrel{-10}{\underline{-10}}$, photoemission spectra are metallic. Thus the need of first-principles methods, capable to accurately capture the delicate balance between the many competing interactions in a real material, is particularly evident in such a complex phase diagram. Kohn-Sham (KS) density-functional theory $(\mathrm{DFT}) \stackrel{11}{\Perp}$ band structures in either the local-density approximation (LDA) or the generalized-gradient approximation (GGA) are metallic for $\mathrm{NiS}_{2}{ }^{9}{ }^{9} 12$. On the other hand, the computational cost of the sophisticated dynamical mean-field theory (DMFT) model limited its application to the high-temperature region of the phase dia$\operatorname{gram}(\mathrm{T}=580 \mathrm{~K} \text {, which is above the Néel temperature })^{10}$, where the magnetic transition is lost.

Here instead our goal is to describe simultaneously the electronic and magnetic properties of $\mathrm{NiS}_{2}$ upon Se doping and under pressure. We therefore consider the two end points of the $\mathrm{T}=0 \mathrm{~K}$ phase diagram of $\mathrm{NiS}_{2-x} \mathrm{Se}_{x}$, together with a representative intermediate point (NiSSe). Moreover, we compare $\mathrm{NiS}_{2}$ at ambient pressure and at $\mathrm{P}=9 \mathrm{GPa}$, which is well above the experimental transition pressure $(\mathrm{P}=2.9 \mathrm{GPa})$. We make use of the $a b$ initio many-body GW approximation $\underline{13}$ and of two more approximated (but computationally much cheaper) approaches: GGA $+\mathrm{U}^{14}$ and the exact exchange for correlated electrons (EECE) hybrid functional ${ }^{15}$. In this way, on the one side we provide a coherent understanding of $\mathrm{NiS}_{2}$ and on the other side we can discuss the advantages and the shortcomings of the different methods for their application in correlated materials.

The paper is organised as follows. In Sec. II we briefly review the different theoretical methods that we have employed in the present study, underlying their differences and commonalities. In Sec. III we present the crystal and magnetic structures of the studied compounds and in Sec. IV the computational details. We then discuss our results. In Sec. $\mathrm{V}$ we focus on $\mathrm{NiS}_{2}$. We first show that the role of non-local Fock exchange is essential to 
obtain an insulating density of states (DOS), correcting the GGA metallic result. We then analyse the electronic and magnetic properties obtained with the other advanced band structure methods, concluding that $\mathrm{NiS}_{2}$ is a charge-transfer insulator. We investigate the effects on the electronic and magnetic properties of $\mathrm{NiS}_{2}$ by $\mathrm{Se}$ doping in Sec. VI, and by applying pressure in Sec. VII. We compare the different mechanisms that induce the IMT and the disappearance of the magnetic order. Finally, in Sec. VIII we summarize our results, drawing our conclusions on the electronic and magnetic properties of $\mathrm{NiS}_{2}$, and comparing the performances of the different methods here employed.

\section{METHODS}

The standard model of electronic structure calculations is based on density-functional theory (DFT) ${ }^{11}$ in the Kohn-Sham (KS) formalism $\frac{16}{2}$. In the KS single-particle Schrödinger equation (atomic units are used throughout this section):

$$
\left(-\frac{\nabla^{2}}{2}+V_{s}(\mathbf{r})\right) \varphi_{i}(\mathbf{r})=\epsilon_{i} \varphi_{i}(\mathbf{r})
$$

the KS local potential $V_{s}(\mathbf{r})=V_{\text {ext }}(\mathbf{r})+V_{H}(\mathbf{r})+V_{x c}(\mathbf{r})$ is the sum of the ionic $V_{e x t}$, Hartree $V_{H}$ and exchangecorrelation $V_{x c}$ terms. In the present work, we use the spin-polarized GGA for $V_{x c}$. By construction, the KS orbitals $\varphi_{i}$ yield the ground-state electronic density:

$$
\rho(\mathbf{r})=\sum_{i} f_{i}\left|\varphi_{i}(\mathbf{r})\right|^{2}
$$

where $f_{i}$ is the Fermi occupation of the state $i$ (in our shorthand notation, $i$ represents band, $\mathbf{k}$ point and spin indeces). Additionally, the KS eigenvalues $\epsilon_{i}$ from Eq. (11) are often interpreted as band structure energies. Unfortunately, this approach results in an underestimation of band gaps in semiconductors and insulators $17-19$. Sometimes this band-gap underestimation may lead to a more severe problem: metallic band structures in smallgap insulators. A possible way to overcome this problem, in compounds with partially filled $d$ or $f$ shells, is the LDA $+\mathrm{U}$ approach ${ }^{14,20,21}$ which adds an intra-atomic Hubbard U repulsion term to the LDA (or GGA) functional. This correction acts only on the "correlated" $d$ or $f$ subset of electrons, leading to an orbital-dependent KS potential (contrary to LDA/GGA, which are orbital independent). The hopping parameters of the resulting Hubbard model are taken from LDA/GGA, while the Hubbard $\mathrm{U}$ is either used as adjustable parameter or evaluated according to different recipes ${ }^{22}-24$ (among which the constrained LDA scheme, used here). A double-counting correction has to be added to the $\mathrm{LDA}+\mathrm{U}$ functional ${ }^{21}$ in order to subtract the interaction between $d$ or $f$ electrons that is partially accounted for in LDA/GGA. The effect of the additional Hubbard $\mathrm{U}$ term is to favor integer occupations with respect to LDA/GGA. This gives rise to a band gap opening, when the gap is located between $d$ or $f$ bands that are partially occupied in LDA/GGA.

Actually, DFT is a ground-state theory and KS eigenvalues are formally only Lagrange multipliers with no physical meaning. Instead, the additional and removal energies $E_{i}$, measured e.g. by photoemission experiments, are the poles of the one-particle Green's function $G^{13,18,25}$. These quasiparticle (QP) energies $E_{i}$ can be obtained by solving a more complex Schrödinger-like equation:

$$
\begin{aligned}
\left(-\frac{\nabla^{2}}{2}+V_{e x t}(\mathbf{r})+V_{H}(\mathbf{r})\right) \phi_{i}(\mathbf{r}) & \\
& +\int d \mathbf{r}^{\prime} \Sigma\left(\mathbf{r}, \mathbf{r}^{\prime}, E_{i}\right) \phi_{i}\left(\mathbf{r}^{\prime}\right)=E_{i} \phi_{i}(\mathbf{r})
\end{aligned}
$$

where the self-energy $\Sigma$ is a non-local, energy-dependent and non-hermitian operator. In the GW approximation $(\mathrm{GWA})^{13}$, the self-energy is given by the convolution between the Green's function $G$ and the dynamically screened Coulomb interaction $W$ :

$$
\Sigma\left(\mathbf{r}, \mathbf{r}^{\prime}, \omega\right)=\frac{i}{2 \pi} \int d \omega^{\prime} e^{i \eta \omega^{\prime}} G\left(\mathbf{r}, \mathbf{r}^{\prime}, \omega+\omega^{\prime}\right) W\left(\mathbf{r}, \mathbf{r}^{\prime}, \omega^{\prime}\right),
$$

where $\eta \rightarrow 0^{+}$and $W(\omega)=\epsilon^{-1}(\omega) v$, with $v$ the Coulomb interaction and $\epsilon$ the dielectric function.

In standard GW calculations $18,25,26$, the QP energies $E_{i}$ are evaluated as first-order perturbative self-energy corrections to the KS eigenvalues $\epsilon_{i}$. This scheme, often named one-shot $\mathrm{G}_{0} \mathrm{~W}_{0}$, is questionable whenever QP wavefunctions $\phi_{i}$ cannot be safely approximated by the $\mathrm{KS}$ orbitals $\varphi_{i}$, and KS results are a bad zeroth-order approximation. This is the case of $\mathrm{NiS}_{2}$. Therefore, in the present case we obtain QP wavefunctions and energies from a self-consistent (sc) solution of the QP equation (31) in the COHSEX approximation ${ }^{13}$. In such a way the final result is independent of the quality of the KS starting point. COHSEX is a static approximation to the GW self-energy with an instantaneous screened interaction $W$. It consists of statically screened exchange (SEX) term plus a Coulomb hole $(\mathrm{COH})$ term, which represents the induced classical potential due to an extra point charge. The sc-COHSEX result is then used as input of a final $\mathrm{G}_{0} \mathrm{~W}_{0}$ calculation, which further takes into account dynamical correlation effects (neglected in the COHSEX approximation). This sc-COHSEX + GW approach $^{27}$ has been proved to accurately describe correlated transition-metal oxides, in both their insulating and metallic phases 28,29 .

The GWA accounts for both Fock exchange and dynamical correlation, which is treated at the level of the random-phase approximation for the dynamical screening of the Coulomb interaction. When the dynamical screening of the Coulomb interaction is neglected by setting $\epsilon^{-1}=1$ in (4), the Hartree-Fock (HF) approximation 
(a)

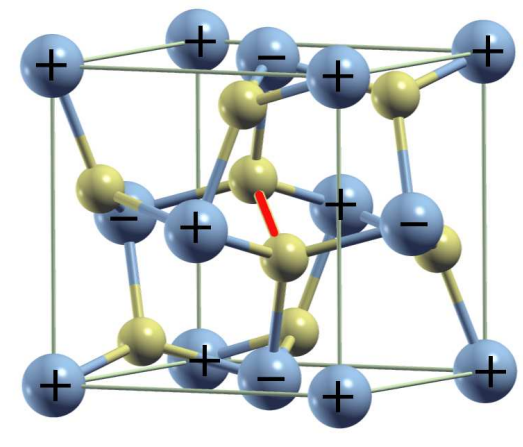

(b)

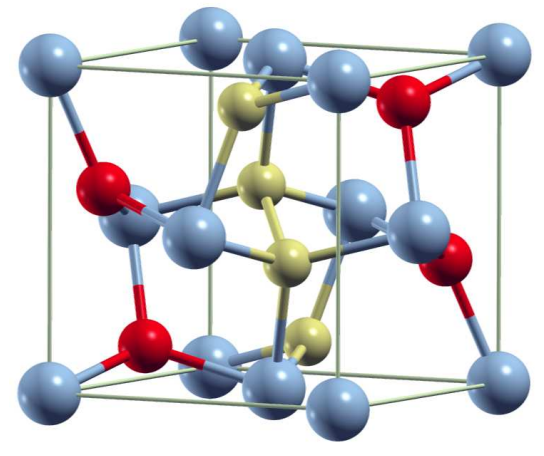

(c)

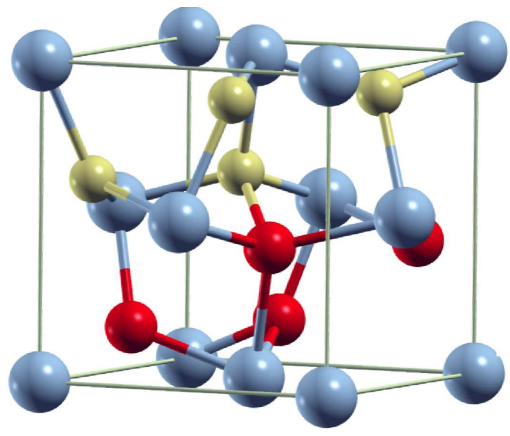

FIG. 1. (Color online) Structures of (a) $\mathrm{NiS}_{2}$, (b) NiSSe (I), and (c) NiSSe (II) (see main text). Ni atoms are light blue, S atoms gold and $\mathrm{Se}$ atoms red balls. In the $\mathrm{NiS}_{2}$ structure, we have highlighted the $\mathrm{S}-\mathrm{S}$ dimer together with the antiferromagnetic order ( + and - correspond to spin up and down local moments, respectively). $\mathrm{NiSe}_{2}$ has the same structure as $\mathrm{NiS}_{2}$, with $\mathrm{Se}$ replacing S atoms.

is retrieved and Eq. (3) reduces to the HF equation. Differently from KS, in HF the exchange term is a non-local operator (the Fock potential) and the HF eigenvalues can be formally interpreted as (approximated) additional and removal energies (i.e. the Koopmans theorem holds in $\mathrm{HF})$.

While local and semilocal approximations, like LDA or GGA, to the local KS $V_{x c}$ potential underestimate band gaps in solids, the HF approximation leads to their overestimation. Adopting a pragmatic point of view, hybrid functionals mix the local KS potential with a portion of non-local Fock exchange. In practice, this mixing interpolates between LDA/GGA and HF results, often allowing for a better agreement with the experiment. In fact, one of the most relevant problems of LDA/GGA is the self-interaction error (SIE), which especially affects localised electrons from $d$ and $f$ shells. In HF the SIE is zero. Thus, recently Tran et al $\stackrel{15}{=}$ proposed to correct partially for the LDA/GGA SIE by adding a fraction $\alpha$ of the non-local Fock term acting only on $d$ and $f$ electrons within the atomic spheres. This scheme is named as "exact exchange for correlated electrons" (EECE) hybrid functiona 15 and has been firstly implemented in the Wien $2 \mathrm{k}$ code ${ }^{30}$. The resulting approximation is an orbital-dependent potential, as within the $\mathrm{LDA}+\mathrm{U}$ scheme. Thus, EECE is a variant of other hybrid functionals commonly used in solid state electronic structure calculations, like PBE0 and HSE 31 , which instead treat all electrons on equal footing.

Both EECE and LDA+U functionals can be derived from a static and local ("on-site") approximation to the dynamically screened Coulomb interaction $W^{20}$, which enters the GW self-energy in convolution with the one-particle Green's function $G$. But, contrary to the GW self-energy, EECE and $\mathrm{LDA}+\mathrm{U}$ corrections to LDA/GGA act directly only on "correlated" Ni $3 d$ states and depend on a parameter. In $\mathrm{LDA}+\mathrm{U}$ it is the onsite Hubbard $U$ and in EECE the fraction $\alpha$ of the nonlocal Fock term that is mixed inside the atomic spheres with the LDA KS exchange-correlation potential (thus $\alpha$ can be understood as a static effective screening of the Coulomb interaction).

\section{CRYSTAL AND MAGNETIC STRUCTURES}

In the present work, we focus on some end points of the phase diagram: $\mathrm{NiS}_{2}, \mathrm{NiSSe}, \mathrm{NiSe}_{2}$ and non-magnetic $\mathrm{NiS}_{2}$ under pressure (in the latter we have neglected a small monoclinic distortion ${ }^{3}$ ). $\mathrm{NiS}_{2}$ in antiferromagnetic insulator, NiSSe an antiferromagnetic metal and $\mathrm{NiSe}_{2}$ a paramagnetic metal. The common cubic pyrite structure of the different compounds is best described in terms of a $\mathrm{NaCl}$ structure with the transition metal in one sublattice and the center of mass of the chalcogen dimers in the other (see Fig. 1). When available, we have used the experimental crystal structures 32 .

We have considered the experimental antiferromagnetic alignment of type I (M1 structure) [see Fig. 1(a)], which has a $\mathbf{Q}=(1,0,0)$ wavevector and orders below $T_{1}=37-54 \mathrm{~K}$ (according to the sample). Experimentally, three different magnetic orderings coexist at low temperature (below $T_{2} \sim 30 \mathrm{~K}$ ) in $\mathrm{NiS}_{2}{ }_{2}^{3.33-35}$ (besides type-I antiferromagnetism, type-II antiferromagnetism and a weak ferromagnetism also occur), giving rise to a complicated magnetic structure. In the M1 structure, the experimental magnetic moment is $1.0 \mu_{B} 33$ (which is smaller than expected from a pure atomic $d^{8}$ configuration).

As found also in the experiments ${ }^{5}$, for NiSSe we have studied two different configurations: the first with $\mathrm{S}_{2}$ and $\mathrm{Se}_{2}$ dimers, the second with mixed S-Se pairs [see Fig. 1(b)-(c)]. In this case, internal positions have been obtained by force minimisation in GGA.

The lattice parameter is expanded by Se alloying (from $5.69 \AA$ in $\mathrm{NiS}_{2}$ to $5.96 \AA$ in $\mathrm{NiSe}_{2}{ }^{32}$ ), while it is compressed under pressure. For $\mathrm{NiS}_{2}$ at $9 \mathrm{GPa}$ and NiSSe we have used the theoretical values: $5.49 \AA$ and $5.87 \AA$, respectively. Moreover, while in $\mathrm{NiS}_{2}$ under pressure the $\mathrm{S}_{2}$ dimer length hardly varies around $2.10 \AA$ (from $2.17 \AA$ at ambient pressure), in $\mathrm{NiSe}_{2}$ the $\mathrm{Se}_{2}$ dimer distance is 
much longer, $2.36 \AA$. Turning to NiSSe, we observe that in NiSSe (I) the S-S and Se-Se distances are comparable to the dimer lengths of the pristine materials. In NiSSe (II), on the other hand, the S-Se distance corresponds to an averaged value of $2.3 \AA$. Already from these structural considerations one may expect that the IMT follows two distinct routes with Se doping or under pressure ${ }^{9.10,36}$, as we will discuss in the following.

\section{COMPUTATIONAL DETAILS}

For GGA, GGA+U and EECE calculations, we have used the full-potential Wien $2 \mathrm{k}$ code $\mathrm{e}^{30}$. For GW and HF calculations, we have used the plane-wave abinit code 37 . In particular, the perfect agreement between results obtained, at the same level of approximation, with Wien $2 \mathrm{k}$ and abinit has allowed us to validate the pseudopotentials employed in the plane-wave calculations.

In calculations with Wien $2 \mathrm{k}$ we have used a up to $7 \times 7 \times 7$ mesh of $\mathrm{k}$ points, the following atomic sphere radii $R_{M T}$ : Ni 2.31 - 2.42 a.u., S 1.98 a.u., Se 2.07 a.u., a plane-wave cutoff $R_{M T} K_{\max }=7$ and 168 local orbitals $(L O)$. The largest vector in the Fourier expansion of the charge density $G_{\max }$ has been set to 12 Ry. In GGA+U we have obtained U from a constrained GGA calculation. Assuming a $\mathrm{Ni}^{8}$ configuration, we have found $U_{\text {eff }}=U-J=6.39 \mathrm{eV}$ for $\mathrm{NiS}_{2}, U_{\text {eff }}=5.89 \mathrm{eV}$ for NiSSe, and $U_{\text {eff }}=4.94 \mathrm{eV}$ for $\mathrm{NiSe}_{2}$. As for the double counting correction, we have compared the fully localized limit (FLL) 14 and the around mean-field treatment $(\mathrm{AMF})^{38}$. In EECE we have tuned the $\alpha$ parameter to reproduce the experimental optical gap in $\mathrm{NiS}_{2}$, finding the value $\alpha=0.2$.

For the GW and HF calculations, we have used Troullier-Martins ${ }^{39}$ norm-conserving pseudopotentials with Ni $3 s$ and $3 p$ semicore states in valence and a $110 \mathrm{Ha}$ energy cutoff in the plane-wave basis expansion. 36 occupied and 24 unoccupied bands in $4 \times 4 \times 4$ k-point grid have been calculated self-consistently in the COHSEX approximation before the final perturbative GW correction. For sake of consistency, in the following, in all the DOS obtained in the different approximations we will plot only those bands that have been calculated self-consistently in the sc-COHSEX+GW scheme. scCOHSEX calculations have been checked starting from either KS or HF obtaining the same results. We have used 240 bands for $W$ and for the GW self-energy and $1800 \mathbf{G}$ vectors for the dielectric matrix $\left(900\right.$ in $\left.\mathrm{NiS}_{2}\right)$ in the Godby-Needs plasmon-pole approximation ${ }^{40}$.

\section{V. $\mathrm{NIS}_{2}$}

\section{A. The role of non-local exchange}

We start our investigation by addressing the following issue. Why is $\mathrm{NiS}_{2}$ metallic in GGA (while it should have

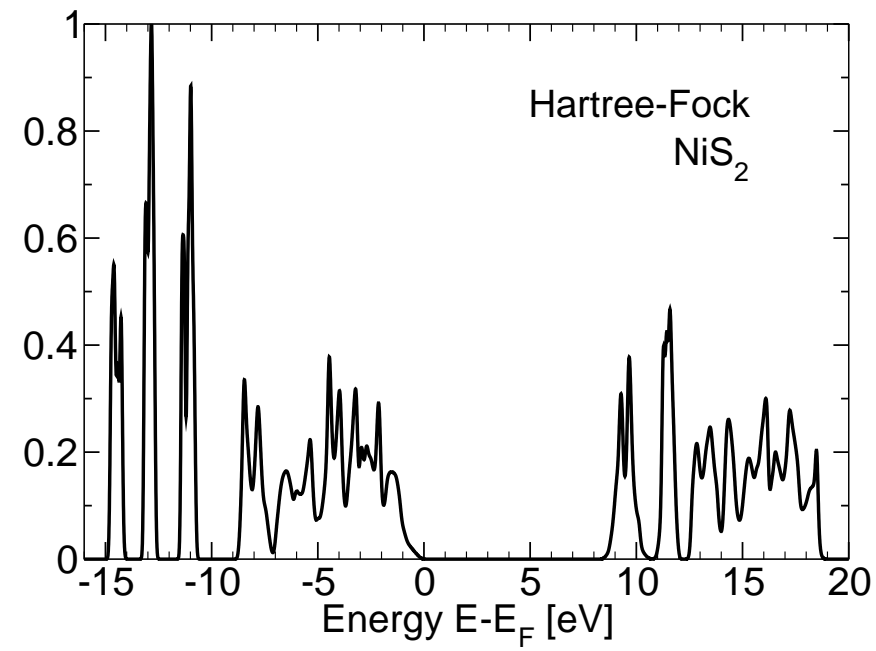

FIG. 2. Hartree-Fock density of states for $\mathrm{NiS}_{2}$. Here, and in the following, we have set the top-valence energy to $E_{F}$ and normalised the DOS to the intensity of the highest peak.

a finite band gap)? Is this a consequence of an inadequate treatment of electronic correlation at the GGA level or, rather, is this finding related to the usual GGA underestimation of band gaps? To answer to these questions, we compare the density of states (DOS) obtained within KS DFT using GGA (see Fig. 3) and within the HF approximation (see Fig. 2). The latter, by definition, neglects completely electronic correlation. Thus, it establishes a clean reference to discuss the effects of electronic correlation. This is a much more difficult task using GGA as the uncorrelated reference, since KS eigenvalues are not rigorous QP energies and LDA/GGA include (approximately) also correlation effects.

We find that, contrary to GGA, the HF DOS is insulating for $\mathrm{NiS}_{2}$, with a very large indirect band gap of $8.3 \mathrm{eV}$. We therefore conclude that $\mathrm{NiS}_{2}$ is insulating also without invoking the effect of strong electronic correlation $\underline{41}$. In fact, the band gap opening in HF is an effect of the non-local Fock exchange. Since the HF gap is much larger than the experimental one, the effect of correlation beyond HF should be instead to reduce the very large HF gap.

Above the Néel temperature, $\mathrm{NiS}_{2}$ remains insulating, while becoming paramagnetic. Whereas a spinunpolarized HF calculation would wrongly give a metal, a proper treatment of the (disordered) local moments 42 would be very helpful in establishing the role of electronic correlation also above the Néel temperature $\underline{43}$.

In GGA the Kohn-Sham orbitals are too much delocalized $\underline{44}$, and for this reason $\mathrm{NiS}_{2}$ is metallic in GGA. In fact, the Fock non-local exchange results in their localisation (which is actually exaggerated in $\mathrm{HF}^{44}$ ). Thus, all the methods that, in a way or in another, are able to mimick this localisation effect can be used to improve over the GGA metallic results. In the following, we discuss three of such methods: the many-body GWA, 

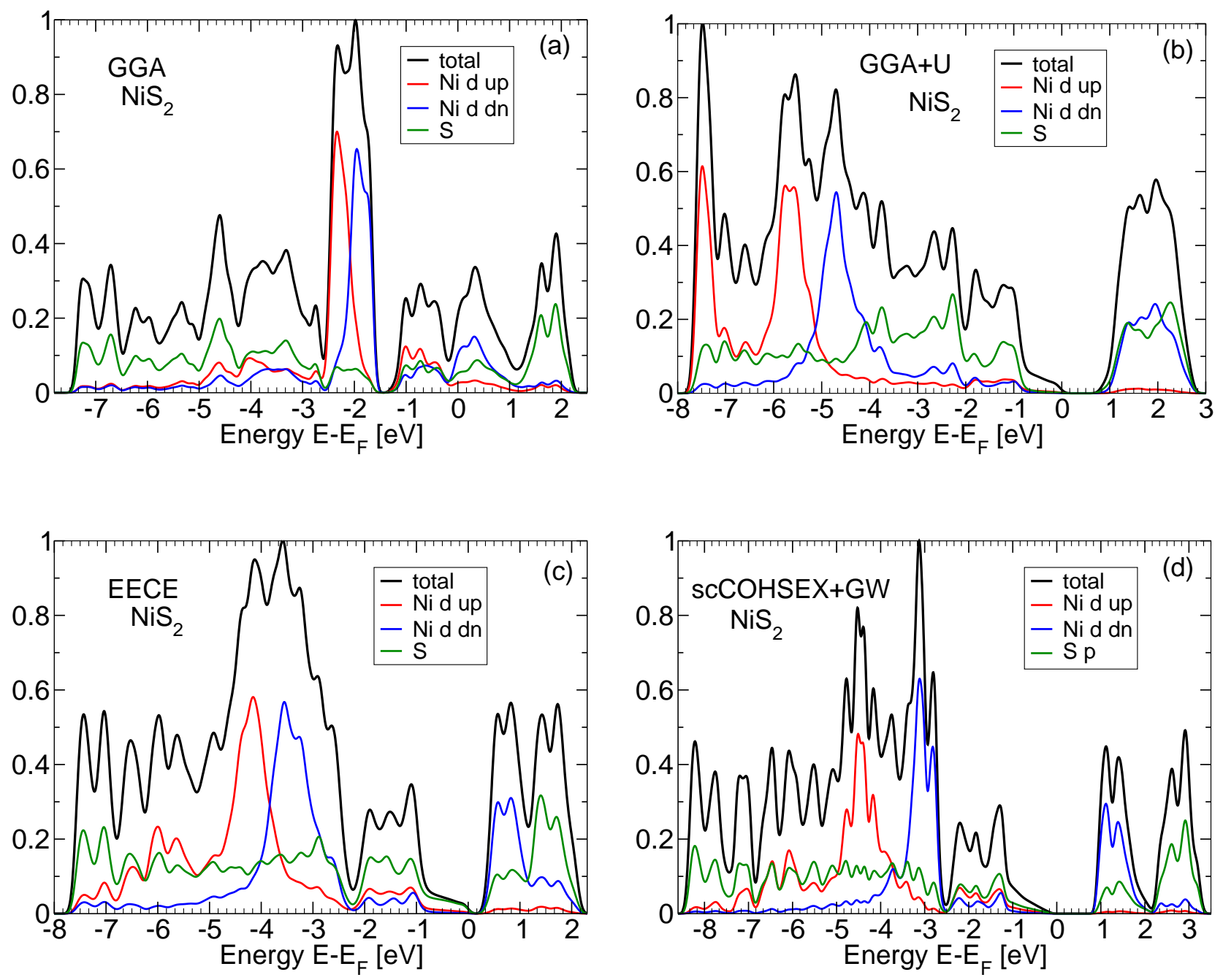

FIG. 3. (Color online) Total density of states of $\mathrm{NiS}_{2}$ and contributions of the Ni $3 d$ and $\mathrm{S} 3 p$ states, calculated in (a) GGA, (b) GGA+U, (c) EECE and (d) sc-COHSEX+GW.

the hybrid EECE and the GGA+U functionals.

\section{B. $\mathrm{NiS}_{2}$ : a charge-transfer insulator}

The discrepancy with respect to experiment is too large to use HF results to analyse the electronic properties of $\mathrm{NiS}_{2}$, which is the goal of the present section. Electronic correlation, and in particular the screening of the Coulomb interaction, indeed has to be taken into account. Here, by using the other advanced electronic structure methods, we address the following questions: What is the insulating nature of $\mathrm{NiS}_{2}$ ? Specifically, among which states does the gap open? How do the DOS here obtained compare with available experimental spectra (although keeping in mind the experimental limitations in the bulk sensitivity) and previous theoretical results?

While $\mathrm{NiS}_{2}$ is metallic in GGA, it correctly turns out to be an antiferromagnetic insulator in all the other ap- proaches (see Fig. 3). In GGA the magnetic moment is $0.7 \mu_{B}$, slightly underestimating the experimental value $1.0 \mu_{B} 33$. In sc-COHSEX $+\mathrm{GW}$ it is enhanced up to 1.3 $\mu_{B}$ and this result is connected to an overestimation of the fundamental band gap that amounts to $0.9 \mathrm{eV}$. In EECE with $\alpha=0.2$ the magnetic moment is $1.4 \mu_{B}$, but the band gap is $0.3 \mathrm{eV}$. This increase of the local moment, going from GGA to EECE or sc-COHSEX+GW, is accompanied by an increase of the exchange splitting of the Ni $d$ states, which are also shifted to higher binding energies.

Besides the different value of the gap, EECE and scCOHSEX $+\mathrm{GW}$ have pretty similar DOS. The presence of $\mathrm{S}_{2}^{-2}$ dimer leads to a large splitting of $3 p$ states into the bonding $p p \sigma$ and anti-bonding $p p \sigma^{*}$ states [which form in itself a double-peak structure, respectively at the bottom and the top of the DOS shown in Fig. 3(c)-(d)]. The $p p \sigma-p p \sigma^{*}$ splitting, and thus the total valence bandwidth, is larger in EECE and sc-COHSEX+GW than in GGA. Inside the $p p \sigma-p p \sigma^{*}$ gap, Ni $3 d$ and S $3 p(p p \pi)$ 


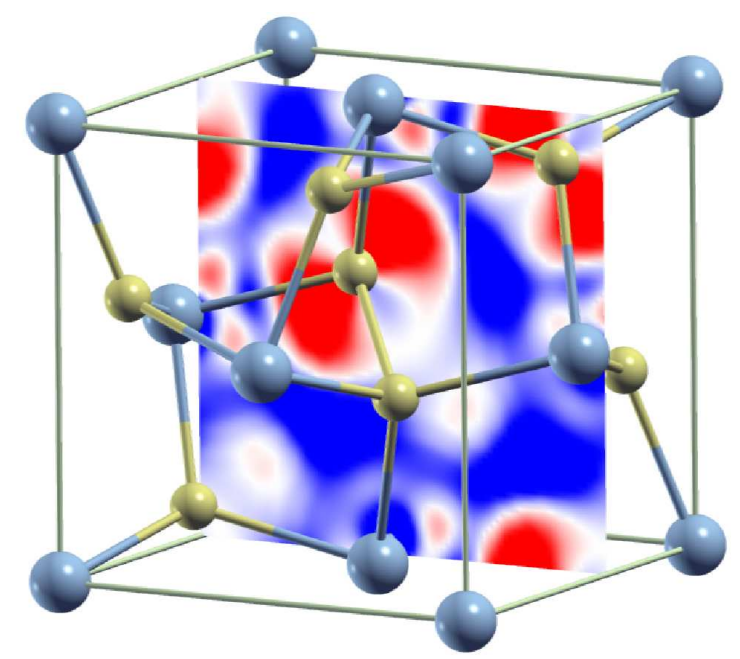

FIG. 4. (Color online) Planar cut of the difference between the quasiparticle electronic charge calculated in scCOHSEX + GW $\left(\rho_{Q P}\right)$ and the Kohn-Sham density $\left(\rho_{K S}\right)$. In red (blue) positive (negative) variations of $\left(\rho_{Q P}-\rho_{K S}\right) / \rho_{K S}$. Gold (light blue) balls are $\mathrm{S}(\mathrm{Ni})$ atoms.

states are highly hybridized. In agreement with resonant photoemission experiments 45 , S $3 p$ states dominate at the highest valence states and $\mathrm{Ni} 3 d$ at the bottom of conduction. Therefore, according to the Zaanen-Sawatzky-Allen classification of transition-metal compounds $\frac{46}{}, \mathrm{NiS}_{2}$ behaves as a charge-transfer insulator.

Also the LDA+DMFT calculation 10 for the hightemperature paramagnetic phase obtained similar results. They differ mainly in the relative amount of $\mathrm{Ni}$ $3 d$ and $\mathrm{S} 3 p$ states that contribute to the bands around the fundamental gap. In fact, the gap similarly opens between highly hybridized Ni-S states. However, both the topmost valence and the bottom conduction bands are there mainly $\mathrm{Ni} 3 d$ (with a larger $\mathrm{S} 3 p$ contribution in the latter). Moreover, in LDA+DMFT an incoherent upper Hubbard band is located at higher energies, which confirms that $\mathrm{NiS}_{2}$ is not a Mott-Hubbard insulator (here, all the incoherent features, including the satellites found in the experiment at the bottom of the valence band ${ }^{5}$ cannot be addressed by a description of the electronic structure based on a quasiparticle DOS).

Overall, the prominent peak of $\mathrm{Ni} 3 d$ states and the other hybridized Ni-S structures at higher binding energies in the valence band are in good correspondence with experimental x-ray photoemission spectra ${ }^{5,47,48}$. Moreover, the two double peaks of the empty states match the double structure measured in bremsstrahlung isochromat spectroscopy ${ }^{6,47}$. The presence of $\mathrm{S} p$ states in the conduction band has been confirmed by X-ray absorption spectroscopy measurements at the $\mathrm{K}$ edge of sulfur ${ }^{10}$.

Instead, in GGA+U (FLL) [see Fig. [3(b)], where the magnetic moment is $1.5 \mu_{B}$, the DOS is quite different: Ni $3 d$ states are shifted to too low energies, forming a triple peak structure at the lower band edge (similarly to
HF, see Fig. 2), S $3 p$ states are homogeneously spread over the whole energy range, and there is only one broad peak for the empty states.

In Fig. 4 we analyse an example of the correction of the LDA/GGA delocalisation error, which is at the origin of the metallic LDA/GGA DOS. We compare the Kohn-Sham charge density $\rho_{K S}(\mathbf{r})$ and the quasiparticle $\rho_{Q P}(\mathbf{r})$ calculated in sc-COHSEX $+\mathrm{GW}$. Blue regions in the planar cut correspond to depletion of QP charge density $\rho_{Q P}$ with respect to $\rho_{K S}$, while red regions correspond to increased charge density going from $\rho_{K S}$ to $\rho_{Q P}$. We find, indeed, that in sc-COHSEX $+\mathrm{GW}$ the charge localises around the $\mathrm{S}$ atoms of the dimers, moving there from the interstial regions. This is associated to an enhancement of the $p p \sigma-p p \sigma^{*}$ bonding-antibonding splitting with respect to $\mathrm{LDA} / \mathrm{GGA}$.

\section{DOPING WITH SE: THE ROLE OF THE DIMERS}

In the previous section, we have concluded that $\mathrm{NiS}_{2}$ is a charge-transfer insulator. The minimum-energy excitation across the band gap is between (mainly) occupied $\mathrm{S} p$ and (mainly) unoccupied $\mathrm{Ni} d$ states, in a wide region of hybridized $\mathrm{Ni} d-\mathrm{S} p$ states. Moreover, from the previous analysis, one may expect that the large anion dimer bonding-antibonding splitting of $p p \sigma$ states plays a prominent role in determining the electronic properties of $\mathrm{NiS}_{2}$. In the present section, we focus on the effect of this bonding-antibonding splitting in more detail.

In fact, the bonding-antibonding splitting can be tuned by replacing $\mathrm{S}$ with Se. Upon doping with Se, the $p p \sigma-$ $p p \sigma^{*}$ bonding-antibonding splitting is reduced due to the increased Se-Se distance in comparison with $\mathrm{S}$ dimers in $\mathrm{NiS}_{2}$ (this reduction is seen also in the experiments 47 ). From this reduction of the bonding-antibonding splitting, the states that lay inside the large $p p \sigma-p p \sigma^{*}$ gap, namely the Ni $3 d$ states and the S $p p \pi$ states, are more strongly overlapping. In turn, NiSSe and $\mathrm{NiSe}_{2}$ are all metallic.

The DOS in NiSSe and $\mathrm{NiSe}_{2}$ are quite similar (see Figs. 56). The dip at $\mathrm{E}_{\mathrm{F}}$ is more pronounced and the conduction band width is smaller in NiSSe (II) than in NiSSe (I). In GGA and in sc-COHSEX+GW $\mathrm{NiSe}_{2}$ is correctly non-magnetic, and, except for a smaller valence bandwidth in GGA, the DOS is overall the same. A posteriori, this would justify the use of the $\mathrm{G}_{0} \mathrm{~W}_{0}$ perturbative approach. In this case, $\mathrm{KS}$ orbitals turn out to be a rather good approximation to the QP wavefunctions, since the orbitals are more delocalized than in $\mathrm{NiS}_{2}$, and the non-local Fock exchange is strongly damped by the metallic screening.

At the critical doping, in NiSSe, we find an antiferromagnetic order, which can be suppressed by applying pressure. In EECE, instead, the calculated moments never vanish. They are $1.3 \mu_{B}$ in NiSSe and $1.2 \mu_{B}$ in $\mathrm{NiSe}_{2}$. GGA $+\mathrm{U}$ results, on the other hand, depend on the choice of the double-counting term. Using FLL the 

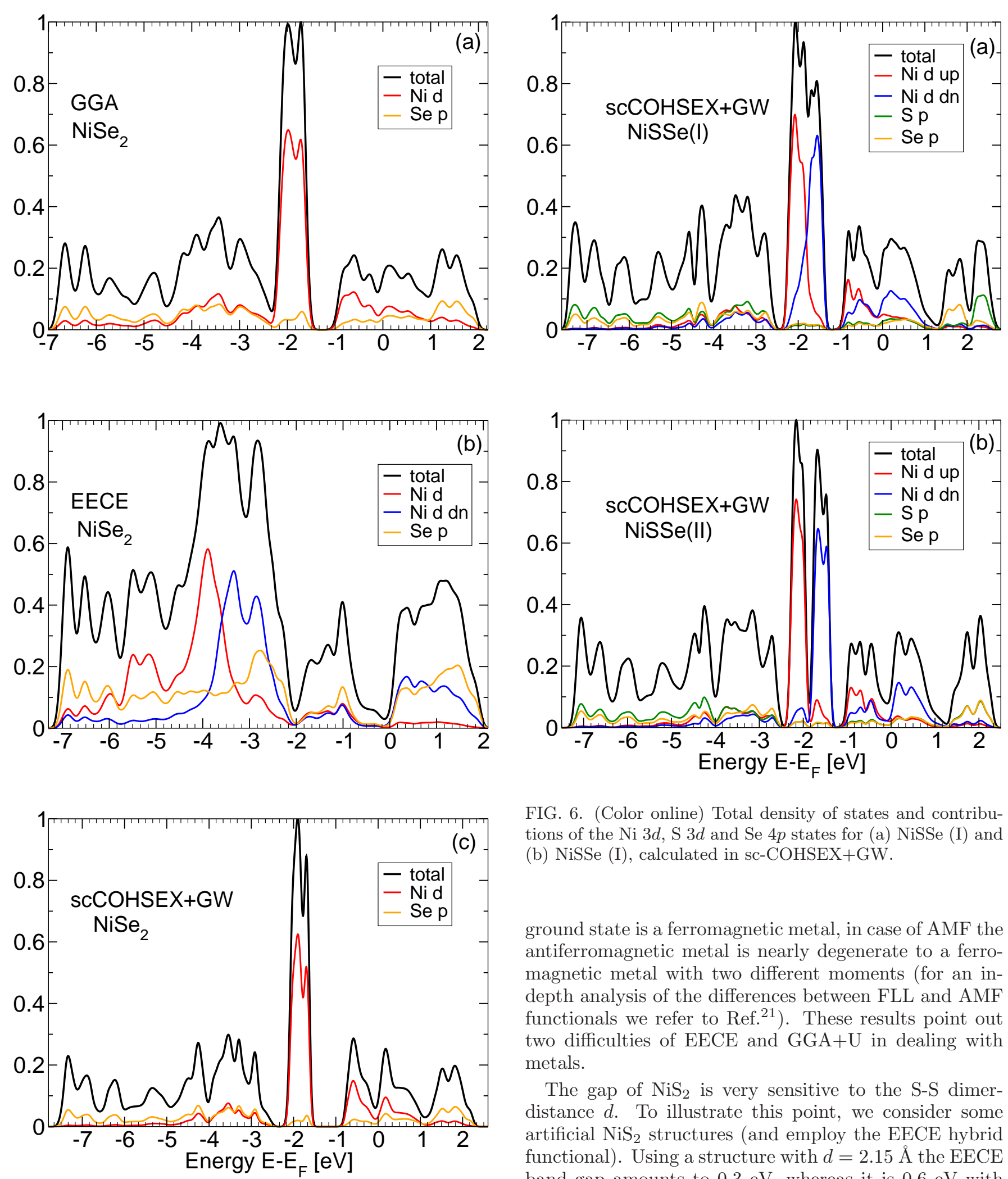

FIG. 6. (Color online) Total density of states and contributions of the Ni $3 d, \mathrm{~S} 3 d$ and Se $4 p$ states for (a) NiSSe (I) and (b) NiSSe (I), calculated in sc-COHSEX+GW.

ground state is a ferromagnetic metal, in case of AMF the antiferromagnetic metal is nearly degenerate to a ferromagnetic metal with two different moments (for an indepth analysis of the differences between FLL and AMF functionals we refer to Ref $\left.\frac{21}{\underline{21}}\right)$. These results point out two difficulties of EECE and GGA+U in dealing with metals.

The gap of $\mathrm{NiS}_{2}$ is very sensitive to the S-S dimerdistance $d$. To illustrate this point, we consider some artificial $\mathrm{NiS}_{2}$ structures (and employ the EECE hybrid functional). Using a structure with $d=2.15 \AA$ the EECE band gap amounts to $0.3 \mathrm{eV}$, whereas it is $0.6 \mathrm{eV}$ with $d=2.10 \AA$. Finally, increasing the dimer distance to $d=2.36 \AA$, i.e. using the structural parameter of $\mathrm{NiSe}_{2}$ but the lattice constant of $\mathrm{NiS}_{2}$, the gap closes. This observation demonstrates that the band gap is related to the dimer distance (instead the magnetic moment is not sensitive to it). When the dimer distance increases, the 
$p p \sigma-p p \sigma^{*}$ splitting decreases, enhancing the overlap of $d$ and $p$ states and thus inducing the closure of the band gap.

In fact, in GGA this bonding-antibonding splitting in $\mathrm{NiS}_{2}$ is underestimated (see Fig. (4) and, similarly to $\mathrm{NiSe}_{2}, \mathrm{NiS}_{2}$ turns out to be metallic in GGA. An analogous underestimation of a dimer bonding-antibonding splitting is at the origin of the absence of a band gap in LDA in other correlated insulators, as for the $\mathrm{V}$ dimers in monoclinic $\mathrm{VO}_{2}$ and the Ti dimers in $\mathrm{Ti}_{2} \mathrm{O}_{3}$. This underestimation is linked to the general LDA/GGA delocalisation error $\underline{\underline{4}}$. When it is amended, also those materials are correctly described as insulators 28,41 . In particular, this correction is found to be an effect of the Fock non-local exchange, as some of us have recently discussed in those materials by making use of HSE hybrid functiona $\stackrel{41}{ }$.

\section{NIS ${ }_{2}$ UNDER PRESSURE: THE ROLE OF CRYSTAL-FIELD SPLITTING}

Applying pressure, the bandwidths are expected to increase. In GGA there is actually a small increase of the widths of the bands around the Fermi energy and the bonding $\mathrm{S} p p \sigma$ bands with respect to $\mathrm{NiS}_{2}$ at ambient pressure, but the main effect is a rigid shift by $-0.5 \mathrm{eV}$ of the prominent $\mathrm{Ni} d$ peak (and a bit more for all the states at higher binding energies), due to an enhanced crystalfield splitting [see Fig. [7(a)]. Hence the lower band edge is about $0.6 \mathrm{eV}$ lower for $a=5.49 \AA$ than for $a=5.69$ $\AA$ and the overall bandwidth is broader. This result is found also in sc-COHSEX+GW calculations, but accompanied also by a larger remodulation of the shape of the DOS [see Fig. 7(c)]. With increasing pressure, both in GGA and in sc-COHSEX+GW the magnetic moment decreases until it disappears. While in case of $\mathrm{NiS}_{2}$ under pressure, GGA+U correctly shows that $\mathrm{NiS}_{2}$ becomes a non-magnetic metal, in EECE, the calculated moments become smaller under pressure but still do not vanish.

Under pressure the dimer distances remain constant, so the electronic and magnetic phase transition can be directly associated to a volume effect, through an increase of the crystal-field splitting and the bandwidths. Thus, this observation highlights the different mechanism that drives the IMT under pressure with respect to that occuring with Se doping. This result is in agreement with previous studies 9.10 .36 , which similarly connected the variation of the anion dimer splitting and the change of the charge-transfer gap in $\mathrm{NiS}_{2-x} \mathrm{Se}_{x}$, and contrasted this mechanism with the change in the bandwidths in $\mathrm{NiS}_{2}$ under pressure. Here we have also remarked that this increase in the bandwidths under pressure (already visible at the GGA level) is accompanied by an enhanced crystal-field splitting (particularly evident at the GWA level).
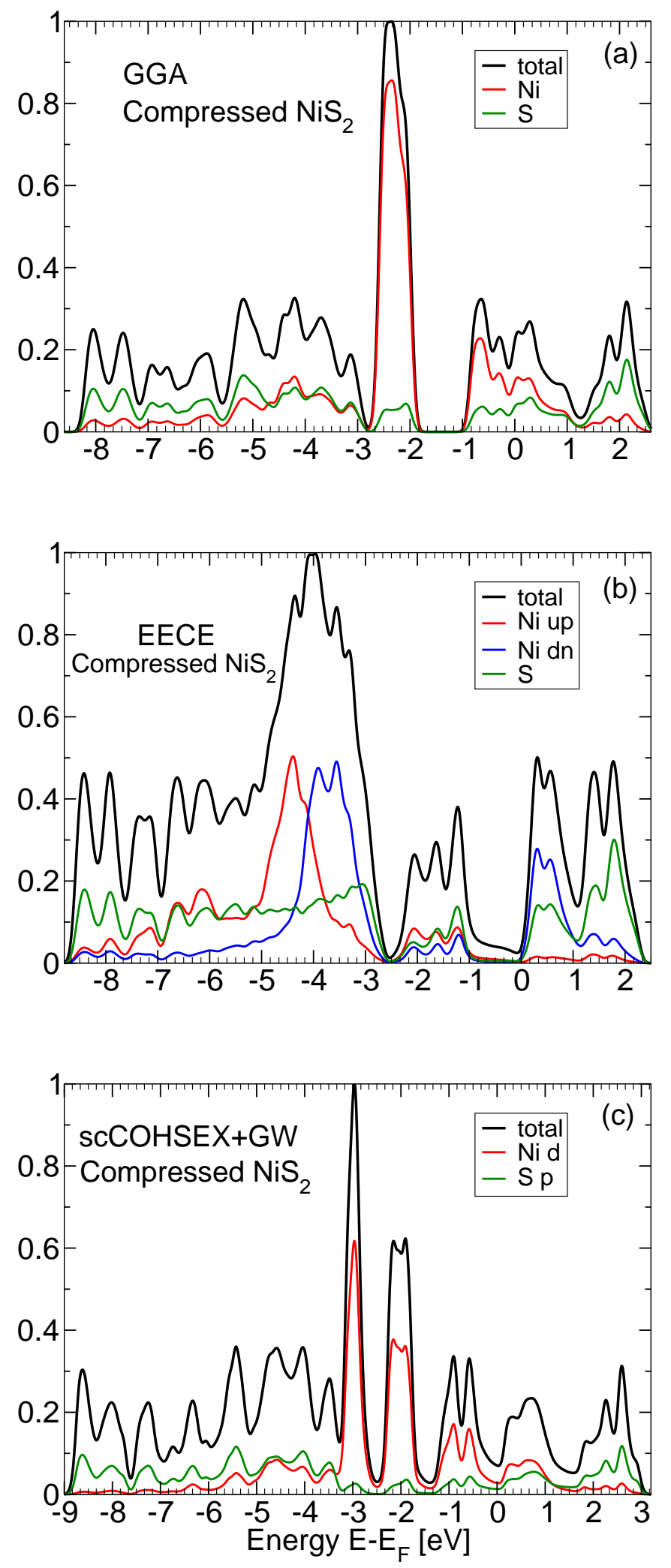

FIG. 7. (Color online) Total density of states of $\mathrm{NiS}_{2}$ under pressure and contributions of the Ni $3 d$ and $\mathrm{S} 3 p$ states, calculated in (a) GGA, (b) GGA+U, and (c) sc-COHSEX+GW. 


\section{CONCLUSIONS}

In summary, we have discussed the electronic and magnetic properties of Se-doped and compressed $\mathrm{NiS}_{2}$ in detail. The results of GGA calculations already allow one to identify the microscopic origin of the metalinsulator and antiferromagnetic-paramagnetic transitions in $\mathrm{NiS}_{2-x} \mathrm{Se}_{x}$. By Se doping, the main effect is the reduction of the bonding-antibonding splitting of $p$ states, which is related to the longer Se dimer distances than for $\mathrm{S}$ dimers. Under pressure, there is an increase of the crystal-field splitting and a bandwidth broadening, both related to a volume effect. In both cases the $\mathrm{NiS}_{2}$ band gap closes, obtaining a metallic state, and the antiferromagnetic order disappears, leading to a paramagnetic state. However, corrections from hybrid EECE and GGA + U density functionals or from the GWA of manybody perturbation theory are necessary to compensate the GGA delocalization error and thus provide the insulating band structure of $\mathrm{NiS}_{2}$. This correction can be understood as mostly due to an effect of non-local exchange. Moreover, although EECE overestimates the magnetic order in the whole phase diagram and parameter-free scCOHSEX $+\mathrm{GW}$ overestimates the fundamental band gap in $\mathrm{NiS}_{2}$, they provide a better description of $\mathrm{NiS}_{2-x} \mathrm{Se}_{x}$ than GGA+U, which turns out to be unreliable for the metallic compounds and in worse agreement with experimental results in insulating $\mathrm{NiS}_{2}$. The sc-COHSEX+GW is the only method always giving the correct qualitative description of the electronic and magnetic properties across the phase diagram. However, the results obtained for $\mathrm{NiS}_{2}$ point out the need of going beyond the present level of approximation, by including vertex corrections beyond the GWA, in order to improve the quantitative agreement with the experimental spectra.

\section{ACKNOWLEDGMENTS}

We acknowledge fruitful discussions with L. Baldassarre, U. Eckern, V. Eyert, J. Kuneš, and Lucia Reining. We have used XCrysDen $\stackrel{49}{\underline{ }}$ for the ball-and-stick representation of the crystal structures. Financial support was provided by the Deutsche Forschungsgemeinschaft (TRR 80), from Spanish MEC (FIS2011-65702-C02-01), ACI-Promociona (ACI2009-1036), Grupos Consolidados UPV/EHU del Gobierno Vasco (IT-319-07), and the European Research Council Advanced Grant DYNamo (ERC-2010-AdG-Proposal No. 267374). Computational time was granted by i2basque.

* matteo.gatti@ehu.es

1 M. Imada, A. Fujimori, and Y. Tokura, Rev. Mod. Phys. 70, 1039 (1998).

2 J. M. Honig and J. Spałek, Chem. Mater. 10, 2910 (1998).

3 Y. Feng, R. Jaramillo, A. Banerjee, J. M. Honig, and T. F. Rosenbaum, Phys. Rev. B 83, 035106 (2011).

4 D. D. Sarma, S. R. Krishnakumar, E. Weschke, C. Schüßler-Langeheine, Chandan Mazumdar, L. Kilian, G. Kaindl, K. Mamiya, S.-I. Fujimori, A. Fujimori, and T. Miyadai, Phys. Rev. B 67, 155112 (2003).

5 S. R. Krishnakumar and D. D. Sarma, Phys. Rev. B 68, 155110 (2003).

6 K. Mamiya, Phys. Rev. B 58, 9611 (1998).

7 T. Thio, J. W. Bennet, and T. R. Thurston, Phys. Rev. B $\mathbf{5 2}, 3555$ (1995).

8 R. L. Kautz, M. S. Dresselhaus, D. Adler, and A. Linz, Phys. Rev. B 6, 2078 (1972).

9 A. Perucchi, C. Marini, M. Valentini, P. Postorino, R. Sopracase, P. Dore, P. Hansmann, O. Jepsen, G. Sangiovanni, A. Toschi, K. Held, D. Topwal, D. D. Sarma, and S. Lupi, Phys. Rev. B 80, 073101 (2009).

10 J. Kuneš, L. Baldassarre, B. Schächner, K. Rabia, C. A. Kuntscher, Dm. M. Korotin, V. I. Anisimov, J. A. McLeod, E. Z. Kurmaev, and A. Moewes, Phys. Rev. B 81, 035122 (2010).

11 P. Hohenberg and W. Kohn, Phys. Rev. 136, B864 (1964).

12 P. Raybaud, J. Hafner, G. Kresse, and H. Toulhoat, J. Phys.: Condens. Matter 9, 11107 (1997); J.-H. Wang, Z. Cheng, J.-L. Brédas, and M. Liu, J. Chem. Phys. 127, 214705 (2007).

13 L. Hedin, Phys. Rev. 139, A796 (1965).

14 V. I. Anisimov, I. V. Solovyev, M. A. Korotin, M. T. Czyźyk, and G. A. Sawatzky, Phys. Rev. B 48, 16929 (1993).

15 F. Tran, P. Blaha, and K. Schwarz, Phys. Rev. B 74, 155108 (2006).

16 W. Kohn and L.J. Sham, Phys. Rev. 140, A1133 (1965).

17 L. J. Sham and M. Schlüter, Phys. Rev. Lett. 51, 1888 (1983).

18 G. Onida, L. Reining, and A. Rubio, Rev. Mod. Phys. 74, 601 (2002).

19 M. van Schilfgaarde, T. Kotani, and S. Faleev, Phys. Rev. Lett. 96, 226402 (2006).

20 V. I. Anisimov, F. Aryasetiawan, and A. I. Lichtenstein, J.Phys.: Condens. Matter 9, 767 (1997).

21 E. R. Ylvisaker, W. E. Pickett, and K. Koepernik, Phys. Rev. B 79, 035103 (2009).

22 I. V. Solovyev, P. H. Dederichs, and V. I. Anisimov, Phys. Rev. B 50, 16861 (1994).

23 M. Cococcioni and S. de Gironcoli, Phys. Rev. B 71, 035105 (2005).

24 M. Springer and F. Aryasetiawan, Phys. Rev. B 57, 4364 (1998).

25 F. Aryasetiawan and O. Gunnarsson, Rep. Prog. Phys. 61, 237 (1998).

26 M. S. Hybersten and S. G. Louie, Phys. Rev. B 34, 5390 (1986).

27 F. Bruneval, N. Vast, and L. Reining, Phys. Rev. B 74, 045102 (2006).

28 M. Gatti, F. Bruneval, V. Olevano, and L. Reining Phys. Rev. Lett. 99, 266402 (2007). 
29 E. Papalazarou, M. Gatti, M. Marsi, V. Brouet, F. Iori, L. Reining, E. Annese, I. Vobornik, F. Offi, A. Fondacaro, S. Huotari, P. Lacovig, O. Tjernberg, N. B. Brookes, M. Sacchi, P. Metcalf, and G. Panaccione, Phys. Rev. B 80, 155115 (2009).

${ }^{30}$ P. Blaha, K. Schwarz, G. Madsen, D. Kvasicka, and J. Luitz, Wien2k: An augmented plane wave + local orbitals program for calculating crystal properties, Vienna University of Technology, 2001.

31 J. Heyd, G. E. Scuseria, and M. Ernzerhof, J. Chem. Phys. 118, 8207 (2003); 124, 219906(E) (2006).

32 P. Kwizera, M. S. Dresselhaus, and D. Adler, Phys. Rev. B 21, 2328 (1980); T. A. Bither, R. J. Bouchard, W. H. Cloud, P. C. Donohue, and W. J. Siemons, Inorg. Chem. 7, 2208 (1968).

${ }^{33}$ T. Miyadai, K. Takizawa, H. Nagata, H. Ito, S. Miyahara, and K. Hirakawa, J. Phys. Soc. Jpn. 38, 115 (1975).

${ }^{34}$ K. Kikuchi, T. Miyadai, H. Itoh, and T. Fukui, J. Phys. Soc. Jpn. 45, 444 (1978); K. Kikuchi, J. Phys. Soc. Jpn. 47484 (1979).

35 M. Matsuura, Y. Endoh, H. Hiraka, K. Yamada, A. S. Mishchenko, N. Nagaosa, and I. V. Solovyev, Phys. Rev. B 68, 094409 (2003).

36 C. Marini, A. Perucchi, D. Chermisi, P. Dore, M. Valentini, D. Topwal, D. D. Sarma, S. Lupi, and P. Postorino Phys. Rev. B 84, 235134 (2011).

37 X. Gonze, G.-M. Rignanese, M. Verstraete, J.-M. Beuken, Y. Pouillon, R. Caracas, F. Jollet, M. Torrent, G. Zerah, M. Mikami, Ph. Ghosez, M. Veithen, J.-Y. Raty, V. Ole- vano, F. Bruneval, L. Reining, R. Godby, G. Onida, D. R. Hamann, and D. C. Allan, Z. Kristallogr. 220, 558 (2005).

38 M. T. Czyzyk and G. A. Sawatzky, Phys. Rev. B 49, 14211 (1994).

39 N. Troullier and J.L. Martins, Phys. Rev. B 43, 1993 (1991).

40 R. W. Godby and R. J. Needs, Phys. Rev. Lett. 62, 1169 (1989).

41 F. Iori, M. Gatti, and A. Rubio, arXiv:1201.3308 (unpublished).

42 D. Hughes, M. Däne, A. Ernst, W. Hergert, M. Lüders, J. B. Staunton, Z. Szotek, and W. M. Temmerman, New J. Phys. 10, 063010 (2008).

43 N. Mott, Metal-Insulator Transitions (Taylor \& Francis, London, 1990), 2nd ed., pags. 138-139.

44 P. Mori-Sanchez, A. J. Cohen, and W. T. Yang, Phys. Rev. Lett. 100, 146401 (2008).

45 A. Fujimori, K. Mamiya, T. Mizokawa, T. Miyadai, T. Sekiguchi, H. Takahashi, N. Môri, and S. Suga, Phys. Rev. B 54, 16329 (1996).

46 J. Zaanen, G. A. Sawatzky, and J. W. Allen, Phys. Rev. Lett. 55, 418 (1985).

47 W. Folkertst, G. A. Sawatzky, C. Haas, R. A. de Groot, and F. U. Hillebrecht, J. Phys. C: Solid State Phys. 20, 4135 (1987).

48 L. Sangaletti, F. Parmigiani, T. Thio and J. W. Bennett Phys. Rev. B 55, 9514 (1997).

49 A. Kokalj, Comp. Mat. Sci. 28, 155 (2003). 